\title{
BUNDLES AND PENCILS OF NETS ON A SURFACE*
}

BY

\author{
ERNEST P. LANE
}

\section{INTRODUCTION}

A net of curves on a surface consists of two one-parameter families of curves such that through each point of the portion of the surface under consideration there passes just one curve of each family, the two tangents at each point being distinct. It is a characteristic property of conjugate nets that at every surface point the two tangents of every conjugate net separate the tangents of the asymptotic net harmonically. In other words, at every point of a surface the cross ratio of the two asymptotic tangents and any two conjugate tangents is -1 . Recent developments in projective differential geometry seem to indicate that these ideas should be generalized.

In the first place, the number -1 may be replaced by any number $r$, and we may then consider the class of all nets on a surface, every one of which has the property that at every surface point its tangents form with the asymptotic tangents the constant cross ratio $r$. In the second place, the asymptotic net may be replaced by an arbitrary fundamental net. We are thus led to introduce the following definition. The class of all nets on a surface, every one of which has the property that at every surface point its two tangents form with the two tangents of a fundamental net the same cross ratio, will be called a bundle of nets. A bundle of nets on a surface is therefore determined by a fundamental net and a number $r$. For example, all conjugate nets on a surface constitute a bundle, for which the fundamental net is the asymptotic net, and for which $r=-1$.

The one-parameter family of conjugate nets on a surface, each of which has the property that, at every surface point, its tangents form with the tangents of a fundamental conjugate net a constant cross ratio, has been called $\dagger$ by Wilczynski a pencil of conjugate nets. This definition can be extended from the bundle of conjugate nets to any bundle of nets. $A$ pencil of nets in a bundle is a one-parameter family of nets in the bundle, each of which has the property that, at every surface point, its two tangents form with the two tangents of a fundamental net of the bundle a constant cross ratio. This cross ratio is different for different nets of the pencil, but for a given

\footnotetext{
* Presented to the Society, December 26, 1924; received by the editors in June, 1925.
}

† Wilczynski, A meri c a n J o u r nal of M a the mat i c s, vol. 42 (1920), p. 216. 
net is the same at every point of the surface. A pencil of nets in a bundle is determined by a fundamental net of the bundle.

\section{BundLes OF NETS}

In order to formulate analytically the definitions which we have introduced, let us suppose that the four homogeneous coördinates $y^{(1)}, \cdots, y^{(4)}$ of an arbitrary point on a surface $S$ are given as analytic functions of two independent variables $u, v$. Then a net of curves on $S$ may be represented by a differential equation of the form

$$
A d u^{2}+2 B d u d v+C d v^{2}=0,
$$

wherein $A, B, C$ are functions of $u, v$ such that $A C-B^{2} \neq 0$. A fundamental net $N_{1}$ being given by equation (1), let us consider a second net $N_{2}$ defined by the equation

$$
L d u^{2}+2 M d u d v+N d v^{2}=0, \quad L N-M^{2} \neq 0 .
$$

We wish to impose on the net $N_{2}$ the condition that at every surface point its two tangents shall form with the two tangents of the net $N_{1}$ a constant cross ratio. So we write down the condition that the two roots of equation (2), regarded as a quadratic in the ratio $d v: d u$, may form with the two roots of equation (1) a cross ratio $r$ which is independent of $u, v$. This condition reduces to

$$
(1-r)^{2}(A N-2 B M+C L)^{2}=4(1+r)^{2}\left(A C-B^{2}\right)\left(L N-M^{2}\right) .
$$

The class of all nets represented on a surface $S$ by an equation of the form (2), whose coefficients satisfy equation (3) with given functions $A, B, C$ and a fixed value of $r$, is a bundle of nets. A bundle of nets on a surface depends therefore on one arbitrary function, and is determined by a net $N_{1}$ and a number $r$.

The symmetry of equation (3) shows that if a net $N_{2}$ belongs to the bundle determined by a net $N_{1}$ and a constant cross ratio $r$, then $N_{1}$ belongs to the bundle determined by $N_{2}$ and the same constant $r$. Interchanging the two component families of $N_{1}$, or of $N_{2}$, changes $r$ to $1 / r$, but leaves equation (3) invariant. If $r=-1$, this equation simplifies into the well known condition that at every surface point the two tangents of $N_{2}$ separate the two tangents of $N_{1}$ harmonically: namely, the vanishing of the harmonic invariant of the binary quadratic differential forms in equations (1) and (2).

If $N_{1}$ is taken for the parametric net on $S$, then $A=C=0, B \neq 0$, and equation (3) takes the form

$$
4 r M^{2}=(1+r)^{2} L N .
$$


We wish to find the differential equation of the most general net $N_{\lambda}$ that belongs to the bundle $B_{r}$ determined by the parametric net and a constant cross ratio $r$. To this end let us consider an arbitrary one-parameter family $F_{\lambda}$ of curves on $S$, distinct from the parametric curves. The equation of such a family $F_{\lambda}$ may be written in the form

$$
d v-\lambda d u=0,
$$

wherein $\lambda$ is an arbitrary function of $u, v$. And let us seek to determine the most general family of curves, represented by the equation

$$
d v-\mu d u=0,
$$

which forms with $F_{\lambda}$ a net of the required kind. Multiplying together the left members of the last two equations and comparing the result with equation (2), we find

$$
L=\lambda \mu, \quad 2 M=-\lambda-\mu, \quad N=1 .
$$

Substituting these functions into equation (4), and solving for $\mu$, we find that we may take $\mu=r \lambda$. Therefore, if the family $F_{\lambda}$, which is represented by equation (5), and the family $F_{r \lambda}$, which is represented by the equation

$$
d v-r \lambda d u=0,
$$

are taken together to form the net $N_{\lambda}$, whose differential equation is

$$
(d v-\lambda d u)(d v-r \lambda d u)=0,
$$

then $N_{\lambda}$ is the most general net that belongs to the bundle $B_{r}$ determined by the parametric net on any surface $S$ and a constant cross ratio $r$.

We might equally well have taken $\mu=\lambda / r$, but this choice is equivalent to interchanging the first and second component families of the net $N_{\lambda}$.

\section{A PENCIL OF NETS IN A BUNDLE}

We propose now to obtain the differential equation of the most general net of the pencil determined by the net $N_{\lambda}$ in the bundle $B_{r}$, and then to find the differential equation of all the curves of this pencil.

The equation of an arbitrary net of the bundle $B_{r}$ may be written in the form

$$
(d v-\mu d u)(d v-r \mu d u)=0 .
$$

We wish to determine the function $\mu$ so that, at every surface point, the two tangents of this net may form with the two tangents of the net $N_{\mathrm{\lambda}}$ a constant cross ratio $c$. Multiplying out the left members of each of equations (7) and (8), and substituting the resulting coefficients into equation (3), 
we find that $\mu$ must be proportional to $\lambda$, so that we have $\mu=\lambda h$, where $h$ is a constant depending on $c$ for its value. Therefore the most general net $N_{\lambda h}$ of the pencil determined by the net $N_{\lambda}$ in the bundle $B_{r},-$ which is itself determined by the parametric net on any surface $S$ and a constant cross ratio $r$,is represented by the equation

$$
(d v-\lambda h d u)(d v-r \lambda h d u)=0,
$$

wherein $h$ is an arbitrary constant. If any other net of this pencil than the net $N_{\lambda}$ is selected as the fundamental net to determine a pencil of nets in the bundle $B_{r}$, the same pencil is obtained as before. Therefore a pencil of nets in a bundle is independent of the one of its nets used to determine it.

The totality of curves contained in all the nets of a pencil in a bundle is a two-parameter family of curves. Such a family is represented in curvilinear coördinates by a differential equation of the second order. If we set either factor of equation (9) equal to zero, solve the resulting equation for $h$, and differentiate totally with respect to $u$, we obtain the differential equation of all the curves contained in the pencil of nets determined by the net $N_{\lambda}$ in the bundle $B_{r}$, namely

$$
\frac{d^{2} v}{d u^{2}}-\frac{\lambda_{u}}{\lambda} \frac{d v}{d u}-\frac{\lambda_{v}}{\lambda}\left(\frac{d v}{d u}\right)^{2}=0 .
$$

If the parametric net is asymptotic, this equation is identical with the equation which we have found* for the curves of a pencil of conjugate nets.

It will be remarked that, although equation (10) appeared as the differential equation of the curves contained in a pencil of nets in the bundle determined by the parametric net and a constant cross ratio $r$, still the equation is independent of $r$. It follows that the curves of a pencil of nets in a bundle are independent of the cross ratio used in defining the bundle. The reason why equation (10) is identical in form with the equation of the curves in a pencil of conjugate nets thus becomes evident.

The curves $u=$ const. are not included among the integral curves of equation (10). If we wish to include them, we may write the equation in a more general and more symmetrical form by supposing that $u$ and $v$ are functions of a parameter $t$ along an integral curve. Equation (10) then becomes

$$
u^{\prime} v^{\prime \prime}-u^{\prime \prime} v^{\prime}-\frac{\lambda_{u}}{\lambda} u^{\prime 2} v^{\prime}-\frac{\lambda_{v}}{\lambda} u^{\prime} v^{\prime 2}=0
$$

wherein accents denote differentiation with respect to $t$.

* Lane, A general theory of conjugate nets, these Transactions, vol. 23 (1922), p. 289. 


\section{Systems $G$}

It is convenient to introduce here the notion of a system $G$ of curves on a surface, by means of the following definition. A system $G$ of curves on a surface $S$ consists of three distinct one-parameter families of curves together with the curves of all the one-parameter families each of which has the property that, at every surface point, its tangent and the three tangents belonging to the three fundamental families form a constant cross ratio. If two of the fundamental families of a system $G$ constitute the parametric net on $S$, and if the third family is $F_{\lambda}$, represented by equation (5), then the family $F_{r \lambda}$, represented by equation (6), is a family of curves belonging to the system $G$. Therefore all the curves of the system $G$ determined by the parametric net and $F_{\lambda}$ are integral curves of equation $\left(10^{\prime}\right)$. Conversely, every equation of the form $\left(10^{\prime}\right)$ can be integrated once and its integral curves can thus be shown to constitute a system $G$.

If we select any three distinct one-parameter families of curves contained in a system $G$ to determine another system $G$, we find the same system $G$ again. Therefore a system $G$ is independent of the three of its oneparameter families used to determine it.

The curves of a system $G$ constitute a special family of hypergeodesics.* In fact, a family of hypergeodesics can be shown to be a system $G$ if, and only if, the curves of the family can be assembled into a single infinity of one-parameter families of curves such that, when any four of the families are selected, the cross ratio, at every surface point, of the four tangents belonging to these four families is constant.

The single infinity of one-parameter families of curves contained in a system $G$ may be paired so as to form a one-parameter family of nets in various ways. We have seen the families of integral curves equation (10) paired, for example, as in equation (9) to form the nets belonging to a pencil of nets in the bundle $B_{r}$. And in particular, if the parametric net is asymptotic and if $r=-1$, we can pair the families of integral curves of (10) to form the conjugate nets of the pencil which is determined by the conjugate net

$$
d v^{2}-\lambda^{2} d u^{2}=0 .
$$

Any one of these nets can have its equation written in the form

$$
d v^{2}-\lambda^{2} h^{2} d u^{2}=0
$$

* Wilczynski, Some generalizations of geodesics, these Transaction s, vol. 23 (1922), p. 226. 


\section{A genERALIZATION OF CONJUGATE NETS}

The bundle of nets determined on a surface by the asymptotic net and an arbitrary constant cross ratio $r$ is a generalization of the bundle of conjugate nets, reducing to the bundle of conjugate nets if $r=-1$. We are now in position to examine the theory of conjugate nets critically with a view to determining what features of this theory are due to the constancy of the cross ratio of conjugate and asymptotic tangents, and what features depend also on the size of this constant. We shall confine ourselves for the most part, however, to an examination of the ray-point cubic and ray conic, and shall find that the ray-point cubic depends only on the constancy of the cross ratio, whereas the ray conic depends on the size of this constant as well.

In order to define the ray-point cubic, let us consider a surface $S_{y}$ on which the asymptotic net is parametric. Such a surface is an integrating surface of a system of differential equations which can be reduced* to the form

$$
\begin{aligned}
y_{u u}+2 b y_{v}+f y & =0, \\
y_{v v}+2 a^{\prime} y_{u}+g y & =0 .
\end{aligned}
$$

The tangents of the family $F_{\lambda}$ represented on $S_{\nu}$ by the equation

$$
d v-\lambda d u=0
$$

form a congruence. The two focal surfaces of this congruence are $S_{y}$ and another surface which we may denote by $S_{\sigma}$. The tangent at a point $P_{y}$ on $S_{y}$ of the curve of $F_{\lambda}$ through $P_{y}$ touches $S_{\sigma}$ at a point $\sigma_{\lambda}$ which is called the ray point of $P_{y}$ with respect to $F_{\lambda}$. The coördinates of the point $\sigma_{\lambda}$ are given $\dagger$ by the formula

$$
\sigma_{\lambda}=\rho+\lambda \sigma+\frac{1}{\lambda}\left(a^{\prime} \lambda^{3}+b\right) y,
$$

wherein we have placed

$$
\rho=y_{u}+\frac{1}{2} \frac{\lambda_{u}}{\lambda} y, \quad \sigma=y_{v}-\frac{1}{2} \frac{\lambda_{v}}{\lambda} y .
$$

The coördinates of the ray point $\sigma_{r \lambda}$ of $P_{y}$ with respect to the family $F_{r \lambda}$ represented by equation (6) are obtained by replacing $\lambda$ by $r \lambda$ in formula (14), with the result

$$
\sigma_{r \lambda}=\rho+r \lambda \sigma-\frac{1}{r \lambda}\left(a^{\prime} r^{3} \lambda^{3}+b\right) y .
$$

* Wilczynski, First Memoir, these Transactions, vol. 8 (1907), p. 233.

† Green, Memoir on the general theory of surfaces, these T r a n s a c $t$ i o n s, vol. 20 (1919),p. 131. 
If we replace $\lambda$ by $\lambda h$ in formulas (14) and (16), we obtain the ray points $\sigma_{\lambda \hbar}$ and $\sigma_{r \lambda h}$ of the point $P_{y}$ with respect to the first and second component families of the net $N_{\lambda h}$ represented by equation (9). If we use the points $y, \rho, \sigma$ in the tangent plane of $S_{y}$ at $P_{y}$ as the vertices of a triangle of reference for a local coördinate system, with suitably chosen unit point, the local coördinates of the ray point $\sigma_{\lambda h}$ are given by the following equations:

$$
x_{1}=-\left(a^{\prime} \lambda^{3} h^{3}+b\right), \quad x_{2}=\lambda h, \quad x_{3}=\lambda^{2} h^{2} .
$$

Finally, if we eliminate $h$ from these equations and make the result homogeneous in the usual way, we obtain the equation of the locus of the ray point $\sigma_{\lambda h}$ as the net $N_{\lambda h}$ varies over all the nets of the pencil determined by the net $N_{\lambda}$ in the bundle $B_{r}$, -which is itself determined by the asymptotic net and a constant cross ratio $r$,-namely

$$
a^{\prime} x_{3}^{3}+b x_{2}^{3}+x_{1} x_{2} x_{3}=0 \text {. }
$$

The locus of the ray point $\sigma_{r \lambda h}$ is the same curve. This is the ray-point cubic which we set out to define.

It will be observed that the ray-point cubic (18) is independent of $r$, and is therefore the same as the ray-point cubic of the pencil of conjugate nets determined by the conjugate net (11), which we have studied* elsewhere. This cubic is really determined by the surface $S_{y}$ and the family $F_{\lambda}$. If, however, any other family of the system $G$ determined by the asymptotic net and $F_{\lambda}$ is used to determine a ray-point cubic, the same curve is obtained as before. Therefore there is in the tangent plane of $S_{y}$ at $P_{y}$ just one ray-point cubic associated with the system $G$ determined by the asymptotic net and any family $F_{\lambda}$. The ray point of $P_{y}$ with respect to each family of the system $G$ lies on this curve. This conclusion can also be reached by use of some of Wilczynski's results concerning hypergeodesics.

We shall next consider the ray conic. The ray of a point $P_{y}$ with respect to a net is the line joining the ray points of $P_{y}$ with respect to the two component families of the net. The ray of $P_{y}$ with respect to the conjugate net (12) has coördinates given by the equations

$$
u_{1}=\lambda^{2} h^{2}, \quad u_{2}=a^{\prime} \lambda^{4} h^{4}, \quad u_{3}=b .
$$

Eliminating $h$, we obtain the equation in line coördinates of the envelope of the rays of $P_{y}$ with respect to all the nets of the pencil of conjugate nets determined by the net (11), namely

$$
a^{\prime} b u_{1}^{2}=u_{2} u_{3}
$$

\footnotetext{
- Lane, A general theory of conjugate nets, these Transactions, vol. 23 (1922), p. 290.
} 
This is the ray conic* of the pencil of conjugate nets. The ray (19) touches the ray conic (20) in the point $Q$ whose coördinates are given by the equations

$$
x_{1}=-2 a^{\prime} b \lambda^{2} h^{2}, \quad x_{2}=b, \quad x_{3}=a^{\prime} \lambda^{4} h^{4},
$$

so that the equation of the ray conic in point coördinates is

$$
4 a^{\prime} b x_{2} x_{3}=x_{1}^{2} .
$$

Observations similar to those made in connection with the ray-point cubic show that there is in the tangent plane of $S_{\nu}$ at $P_{\nu}$ just one ray conic associated with the system $G$ determined by the asymptotic net and any family $F_{\lambda}$. The ray of $P_{y}$ with respect to the conjugate net determined by each family of the system $G$ is tangent to this ray conic.

In order to study the pencil of conics touching the asymptotic tangents at the points where the ray conic touches these lines, let us consider again the net $N_{\lambda h}$ represented by equation (9). The first family of this net determines the conjugate net (12), and the ray of $P_{y}$ with respect to this net touches the ray conic (22) at the point $Q$, whose coördinates are given by equation (21). The second family of the net $N_{\lambda h}$ determines also a conjugate net, whose equation is

$$
d v^{2}-r^{2} \lambda^{2} h^{2} d u^{2}=0 .
$$

The ray of $P_{y}$ with respect to this net touches the ray conic (22) at the point $Q_{r}$ whose coördinates are given by the equations

$$
x_{1}=-2 a^{\prime} b r^{2} \lambda^{2} h^{2}, \quad x_{2}=b, \quad x_{3}=a^{\prime} r^{4} \lambda^{4} h^{4} .
$$

The line $l_{r}$ joining the contact points $Q$ and $Q_{r}$ has coördinates which are given by the equations

$$
u_{1}=\left(1+r^{2}\right) \lambda^{2} h^{2}, \quad u_{2}=2 a^{\prime} r^{2} \lambda^{4} h^{4}, \quad u_{3}=2 b .
$$

If we eliminate $h$ from these equations and make the result homogeneous, we obtain the equation of the envelope of the line $l_{r}$ as the net $N_{\lambda h}$ varies over all the nets of the pencil determined by the net $N_{\lambda}$ in the bundle $B_{r}$, namely

$$
4 a^{\prime} b r^{2} u_{1}^{2}=\left(1+r^{2}\right)^{2} u_{2} u_{3}
$$

The line $l_{r}$ touches this conic at the point whose coördinates are given by the equations

$$
x_{1}=-4 a^{\prime} b r^{2} \lambda^{2} h^{2}, \quad x_{2}=b\left(1+r^{2}\right), \quad x_{3}=a^{\prime} r^{2}\left(1+r^{2}\right) \lambda^{4} h^{4},
$$

\footnotetext{
- Lane, loc. cit., p. 293.
} 
so that the equation of the same conic in point coördinates is

$$
16 a^{\prime} b r^{2} x_{2} x_{3}=\left(1+r^{2}\right)^{2} x_{1}^{2}
$$

If $r=-1$, so that the bundle $B_{r}$ is the bundle of conjugate nets, this conic reduces to the ray conic (22). If $r$ is regarded as a parameter, all the conics represented by equation (28) belong to a pencil of conics, every one of which touches each asymptotic tangent at the point where the flex ray* of the ray-point cubic (18) intersects the tangent.

The polar line of the point (27) with respect to the ray conic has coördinates given by the equations

$$
u_{1}=2 r^{2} \lambda^{2} h^{2}, \quad u_{2}=a^{\prime} r^{2}\left(1+r^{2}\right) \lambda^{4} h^{4}, \quad u_{3}=b\left(1+r^{2}\right),
$$

and the coördinates of the intersection points of this line with the ray conic are found to be given by the following formulas:

$$
\begin{aligned}
& x_{1}=-2 a^{\prime} b r\left(1+r^{2}\right)\left[2 r \pm i\left(1-r^{2}\right)\right] \lambda^{2} h^{2}, \\
& x_{2}=b\left[-1+6 r^{2}-r^{4} \pm 4 r i\left(1-r^{2}\right)\right], \\
& x_{3}=a^{\prime} r^{2}\left(1+r^{2}\right)^{2} \lambda^{4} h^{4},
\end{aligned}
$$

wherein $i=\sqrt{-1}$. Therefore, if we suppose for a moment that all the numbers used hitherto have been real, these intersections are imaginary, so that the point (27) is inside the ray conic, and all the conics represented by equation (28) for different values of $r$ are inside the ray conic. Moreover, it can be shown under this assumption that the conics (28) intersect the raypoint cubic in imaginary points only.

As the net $N_{\lambda h}$ varies over all the nets of the pencil determined by the net $N_{\lambda}$ in the bundle $B_{r}$, the line (29) envelopes a conic whose equation in line coördinates is

$$
a^{\prime} b\left(1+r^{2}\right)^{2} u_{1}^{2}=4 r^{2} u_{2} u_{3}
$$

The point of contact of the line (29) with this conic has coördinates given by the equations

$$
x_{1}=-a^{\prime} b\left(1+r^{2}\right) \lambda^{2} h^{2}, \quad x_{2}=b, \quad x_{3}=a^{\prime} r^{2} \lambda^{4} h^{4},
$$

so that the equation of the same conic in point coördinates is

$$
r^{2} x_{1}^{2}=a^{\prime} b\left(1+r^{2}\right)^{2} x_{2} x_{3} .
$$

- Lane, loc. cit., p. 290. 
Of course, the point (31) is the intersection of the line (19), which is tangent to the ray conic at $Q$, and the line

$$
u_{1}=r^{2} \lambda^{2} h^{2}, \quad u_{2}=a^{\prime} r^{4} \lambda^{4} h^{4}, \quad u_{3}=b,
$$

which is tangent to the ray conic at $Q_{r}$. These two lines are the rays of $P_{y}$ with respect to the two conjugate nets determined by the first and second component families of the net $N_{\lambda h}$. If again we suppose for a moment that all numbers used are real unless otherwise designated, the point (31) is outside the ray conic and all the conics represented by equation (32) for different values of $r$ are outside the ray conic, but are in the same pencil as the conics (28). Moreover, each conic (32) can be shown to intersect the ray-point cubic in two real and four imaginary points.

We shall now show how the ray conic may be generalized in still another way. The ray of $P_{y}$ with respect to the net $N_{\lambda h}$ joins the ray points $\sigma_{\lambda h}$ and $\sigma_{r \lambda h}$ of $P_{y}$ with respect to the two component families of the net. The line coördinates of this ray are found, by means of equations (17), to be given by the equations

$$
\begin{aligned}
& u_{1}=r \lambda^{2} h^{2}, \\
& u_{2}=\lambda h\left[-a^{\prime} r^{2} \lambda^{3} h^{3}+b(1+r)\right], \\
& u_{3}=a^{\prime} r(1+r) \lambda^{3} h^{3}-b .
\end{aligned}
$$

If we eliminate $h$ from these equations and make the result homogeneous, we obtain the equation, in line coördinates, of the envelope of the ray of $P_{y}$ with respect to an arbitrary net $N_{\lambda h}$ of the pencil of nets determined by the net $N_{\lambda}$ in the bundle $B_{r}$, as $N_{\lambda h}$ varies over all the nets of this pencil, namely,

$$
\begin{aligned}
& r^{3} u_{2}^{2} u_{3}^{2}+r^{2}(1+r)^{2} u_{1}\left(a^{\prime} u_{2}^{3}+b u_{3}^{3}\right) \\
& \quad+a^{\prime} b r\left(1+r+r^{2}\right)\left(1+4 r+r^{2}\right) u_{1}^{2} u_{2} u_{3}-a^{\prime 2} b^{2}\left(1+r+r^{2}\right)^{3} u_{1}^{4}=0 .
\end{aligned}
$$

This is a curve of class four and order six. It has a double point at $P_{y}$, the double point tangents being the asymptotic tangents.

If $r$ is regarded as a parameter, equation (35) represents a one-parameter family of curves. We shall characterize two particular curves of this family. First, if $1+r=0$, the curve (35) reduces to the ray conic (20) counted twice. Second, if $1+r+r^{2}=0$, the equation (35) reduces to

$$
u_{2}^{2} u_{3}^{2}+u_{1}\left(a^{\prime} u_{2}^{3}+b u_{3}^{3}\right)=0 .
$$

This curve is the only curve of the family which is tangent to the flex ray of the ray-point cubic (18), and it has the flex ray for triple tangent, the contact 
points being the points where the Segre tangents meet the flex ray, namely, the points represented by the equation

$$
a^{\prime} u_{2}^{3}+b u_{3}^{3}=0 .
$$

The curve has no inflexions, four double points, and six cusps.

We shall find it profitable to obtain the parametric equations of the envelope (35) in point coördinates. Any point $\eta$ on the ray of $P_{y}$ with respect to the net $N_{\lambda h}$ is given by

$$
\begin{aligned}
\eta= & a \sigma_{\lambda h}+\beta \sigma_{r \lambda h}=(a+\beta) \rho+(a+\beta r) \lambda h \sigma \\
& -\frac{1}{r \lambda h}\left[a r\left(a^{\prime} \lambda^{3} h^{3}+b\right)+\beta\left(a^{\prime} r^{3} \lambda^{3} h^{3}+b\right)\right] y,
\end{aligned}
$$

where $a, \beta$ are arbitrary functions of $u, v$. As $N_{\lambda h}$ varies over all the nets of the pencil, the point $\eta$ describes a curve. The point $d \eta / d h$ is on the tangent of this curve, and the point $\eta$ will be the point of contact of the ray with its envelope if, and only if, $d \eta / d h$ is a linear combination of $\sigma_{\lambda \lambda}$ and $\sigma_{r \lambda h}$. Imposing this condition on $\eta$, we find

$$
a: \beta=\left(a^{\prime} r^{2} \lambda^{3} h^{3}+b\right):\left(a^{\prime} r \lambda^{3} h^{3}+b\right) \text {. }
$$

Using this ratio in equations (37), we find that the point of contact of the ray with its envelope has local coördinates given by

$$
\begin{aligned}
& x_{1}=-b^{2}(1+r)-2 a^{\prime} b r\left(1+r^{2}\right) \lambda^{3} h^{3}-a^{\prime 2} r^{3}(1+r) \lambda^{6} h^{6}, \\
& x_{2}=r \lambda h\left[2 b+a^{\prime} r(1+r) \lambda^{3} h^{3}\right], \\
& x_{3}=r \lambda^{2} h^{2}\left[b(1+r)+2 a^{\prime} r^{2} \lambda^{3} h^{3}\right] .
\end{aligned}
$$

These are the parametric equations of the envelope in terms of the parameter $h$.

\section{The Segre-DarbouX SYSTEM $G$}

The Segre curves, whose differential equation is

$$
a^{\prime} d v^{3}-b d u^{3}=0,
$$

and the Darboux curves, whose equation is

$$
a^{\prime} d v^{3}+b d u^{3}=0,
$$

play a significant rôle in the projective differential geometry of a curved surface. The six one-parameter families of Segre and Darboux curves belong to a system $G$, which also contains the asymptotic net, and which we shall call the Segre-Darboux system $G$.

We shall now establish two theorems concerning the Segre-Darboux system $G$. The ray of $P_{y}$ with respect to the net $N_{\lambda}$, represented by equa- 
tion (7), joins the ray point $\sigma_{\lambda}$, given by (14), and the ray point $\sigma_{r \lambda}$, given by (16). The point of contact $\eta$ of this ray with its envelope (35) is given by

$$
\eta=\left(a^{\prime} r^{2} \lambda^{3}+b\right) \sigma_{\lambda}+\left(a^{\prime} r \lambda^{3}+b\right) \sigma_{r \lambda} .
$$

If the family $F_{\lambda}$ is any one of the three families of the Segre-Darboux system $G$ for which the differential equation is

$$
a^{\prime} r^{2} d v^{3}+b d u^{3}=0,
$$

then we have

$$
a^{\prime} r^{2} \lambda^{3}+b=0,
$$

and the point of contact $\eta$ coincides with the ray point $\sigma_{r \lambda}$. The family $F_{r \lambda}$ which forms with $F_{\lambda}$ the net $N_{\lambda}$ of the bundle $B_{r}$ is then one of the three families of the Segre-Darboux system $G$ for which the differential equation is

$$
a^{\prime} d v^{3}+b r d u^{3}=0 \text {. }
$$

Therefore each family represented by equation (39) can be associated with a family represented by equation (40) to form a net which belongs to the bundle $B_{r}$, -determined by the asymptotic net and a constant cross ratio $r$,-and which is such that the ray of $P_{y}$ with respect to this net touches the envelope (35) in the ray point of $P_{y}$ with respect to the component family given by equation (40).

Similarly, if the family $F_{\lambda}$ is any one of the three families for which the differential equation is

$$
a^{\prime} r d v^{3}+b d u^{3}=0,
$$

then the point of contact $\eta$ coincides with the ray point $\sigma_{\lambda}$. The family $F_{r \lambda}$ is then one of the families for which the differential equation is

$$
a^{\prime} d v^{3}+b r^{2} d u^{3}=0 \text {. }
$$

Therefore each family represented by equation (41) can be associated with a family represented by equation (42) to form a net which belongs to the bundle $B_{\text {r }}$ and is such that the ray of $P_{y}$ with respect to this net touches the envelope (35) in the ray point of $P_{y}$ with respect to the component family given by equation (41).

\section{BundLes With CONJUgate NeTS FUNDAMENTAL}

The bundle of nets determined on a surface $S_{y}$ by a conjugate net and a constant cross ratio $r$ is analogous to the bundle of conjugate nets, the asymptotic net of the latter being replaced by an arbitrary conjugate net, and -1 being replaced by $r$. In studying such a bundle we shall suppose 
that the fundamental conjugate net is parametric. Then $S_{\gamma}$ is an integrating surface of a system of differential equations of the form*

$$
\begin{aligned}
& y_{u u}=a y_{v v}+b y_{u}+c y_{v}+d y, \\
& y_{u v}=\quad b^{\prime} y_{u}+c^{\prime} y_{v}+d^{\prime} y .
\end{aligned}
$$

The differential equation of the asymptotic net is

$$
a d u^{2}+d v^{2}=0,
$$

and the family conjugate to the family $F_{\lambda}$, represented by (5) in conjugate parameters, is given by the equation

$$
a d u+\lambda d v=0 .
$$

In order to find the ray point of $P_{\nu}$ with respect to the family $F_{\lambda}$, let us observe that the point $y_{u}+\lambda y_{v}$ is on the tangent at $P_{\nu}$ of the curve of $F_{\lambda}$ through $P_{y}$. Any point $Y$, except $P_{y}$, on this tangent is defined by the formula

$$
Y=y_{u}+\lambda y_{v}+K y,
$$

wherein $K$ is a function of $u, v$ which is to be determined so that the point $Y$ shall be the required ray point. We find, on differentiating $Y$ and making use of system (43),

$$
\begin{aligned}
& Y_{u}=a y_{v v}+\left(b+\lambda b^{\prime}+K\right) y_{u}+\left(c+\lambda c^{\prime}+\lambda_{u}\right) y_{v}+\left(d+\lambda d^{\prime}+K_{u}\right) y, \\
& Y_{v}=\lambda y_{v v}+b^{\prime} y_{u}+\left(c^{\prime}+K\right) y_{v}+\left(d^{\prime}+\lambda_{v}+K_{v}\right) y .
\end{aligned}
$$

The expression $\lambda Y_{u}-a Y_{v}$ does not contain $y_{v v}$, and is a linear combination of $y$ and $y_{u}+\lambda y_{v}$ if, and only if, $K$ satisfies the condition

$$
\left(\lambda^{2}+a\right) K=-b^{\prime} \lambda^{3}+\left(c^{\prime}-b\right) \lambda^{2}+\left(c+a b^{\prime}\right) \lambda-a c^{\prime}+\lambda \lambda_{u}-a \lambda_{0} .
$$

Therefore the ray point $\sigma_{\lambda}$ of $P_{y}$ with respect to the family $F_{\lambda}$ is given by the formula

$$
\sigma_{\lambda}=\left(\lambda^{2}+a\right) \rho+\lambda\left(\lambda^{2}+a\right) \sigma+\lambda(Q \lambda+a P) y,
$$

wherein we have placed

$$
P=\frac{c}{a}+2 b^{\prime}-\frac{\lambda_{v}}{\lambda}, Q=2 c^{\prime}-b+\frac{\lambda_{v}}{\lambda},
$$

and $\rho, \sigma$ are the covarients whose definitions are

$$
\rho=y_{u}-c^{\prime} y, \sigma=y_{0}-b^{\prime} y .
$$

* Green, Projective differential geometry of one-parameter families of space curves, A m e r i c a n Journal of Mathematics, vol. 37 (1915) and vol. 38 (1916). 
In fact, the points $\rho$ and $\sigma$ are the ray points of $P_{\nu}$ with respect to the families $v=$ const. and $u=$ const. respectively.

If we replace $\lambda$ by $\lambda h$ in equation (46), we obtain the ray point $\sigma_{\lambda h}$ of $P_{y}$ with respect to the first family of the net $N_{\lambda h}$ represented by equation (9) in conjugate parameters. And if we use the triangle $y, \rho, \sigma$ as a local triangle of reference, with suitably chosen unit point, the local coördinates of $\sigma_{\lambda h}$ are given by the equations

$$
x_{1}=\lambda h(Q \lambda h+a P), \quad x_{2}=\lambda^{2} h^{2}+a, \quad x_{3}=\lambda h\left(\lambda^{2} h^{2}+a\right) .
$$

Eliminating $h$ from these equations, and making the result homogeneous, we obtain

$$
\left(a x_{1}^{2}+x_{3}^{2}\right)\left(4 x_{1}-Q x_{2}-P x_{3}\right)+a Q x_{2}^{3}-3 a P x_{2}^{2} x_{3}-3 Q x_{2} x_{3}^{2}+P x_{3}^{3}=0 .
$$

This is the ray-point cubic of $P_{y}$ with respect to the system $G$ determined by the parametric conjugate net and the family $F_{\lambda}$. It has a node at $P_{y}$, the double point tangents being the asymptotic tangents,

$$
a x_{2}^{2}+x_{3}^{2}=0 .
$$

The cubic, moreover, has three inflexions which lie on the flex ray

$$
4 x_{1}-Q x_{2}-P x_{3}=0,
$$

and each inflexion lies on one of the lines through $P_{y}$ given by

$$
a Q x_{2}^{3}-3 a P x_{2}^{2} x_{3}-3 Q x_{2} x_{3}^{2}+P x^{3}=0 .
$$

It is interesting to compare the cubic (50) with the cubic (18). For this purpose we shall need to have the equations of the two curves referred to the same coördinate system. Let $\left(x_{1}, x_{2}, x_{3}\right)$ be the coördinates of a point referred to the triangle used in deriving equation (18), and let $\left(\bar{x}_{1}, \bar{x}_{2}, \bar{x}_{3}\right)$ be the coördinates of the same point referred to the triangle used in the present section. Moreover, let the parametric conjugate net for system (43) be the conjugate net (11). Then the transformation of coördinates between these two systems is found to be given by the equations

$$
\begin{array}{lll}
x_{1}=2 \lambda \varphi_{v} \psi_{v}\left(\bar{x}_{1}-\mathcal{G}^{\prime} \bar{x}_{2}-\mathfrak{B}^{\prime} \bar{x}_{3}\right), & -\psi_{v} \bar{x}_{2}+\varphi_{v} \bar{x}_{3}, \\
x_{2}= & \lambda\left(\psi_{v} \bar{x}_{2}+\varphi_{v} \bar{x}_{3}\right), \\
x_{3}= &
\end{array}
$$

where $\lambda$ is the function of the asymptotic parameters that appears in equation (11), and $\varphi, \psi$ are solutions of the equations*

$$
\varphi_{u}+\lambda \varphi_{v}=0, \quad \psi_{u}-\lambda \psi_{v}=0,
$$

* Lane, loc. cit., p. 285. 
while $\mathfrak{O}^{\prime}, \mathfrak{C}^{\prime}$ are the invariants usually defined by the formulas

$$
\mathfrak{B}^{\prime}=\frac{1}{8}\left(4 b^{\prime}+2 \frac{c}{a}-\frac{a_{v}}{a}\right), \quad \mathbb{E}^{\prime}=\frac{1}{8}\left(4 c^{\prime}-2 b+\frac{a_{u}}{a}\right) .
$$

By means of the transformation (51) and the relation*

$$
a \varphi_{v}^{2}+\psi_{v}^{2}=0 \text {, }
$$

equation (18) is transformed into

$$
\left(a x_{2}^{2}+x_{3}^{2}\right)\left(x_{1}-\mathbb{C}^{\prime} x_{2}-\mathscr{B}^{\prime} x_{3}\right)+a \mathbb{S}^{\prime} x_{2}^{3}-3 a \mathscr{B}^{\prime} x_{2}^{2} x_{3}-3 \mathbb{C}^{\prime} x_{2} x_{3}^{2}+\mathscr{B}^{\prime} x_{3}^{3}=0,
$$

the dashes being dropped from the result. Comparison of equations (50) and (53) shows that the two curves are the same if, and only if,

$$
P=4 \mathfrak{B}^{\prime}, \quad Q=4 \mathfrak{C}^{\prime} .
$$

These conditions may be reduced, by means of the definitions (47) and (52), to the differential equations

$$
\frac{\lambda_{u}}{\lambda}=\frac{1}{2} \frac{a_{u}}{a}, \frac{\lambda_{v}}{\lambda}=\frac{1}{2} \frac{a_{v}}{a},
$$

of which the most general solution is

$$
\lambda=\text { const. } \sqrt{a} .
$$

Therefore the ray-point cubics (50) and (18) are the same if, and only if, the system $G$ associated with the cubic (50) contains the asymptotic net.

The transformation (51) is useful for many purposes. For example, the equation $x_{1}=0$ of the flex ray of the cubic (18) becomes, in the notation of conjugate nets,

$$
x_{1}-\mathfrak{C}^{\prime} x_{2}-\mathfrak{B}^{\prime} x_{3}=0 .
$$

The equation of the Darboux tangents is, in this notation,

$$
a \mathbb{S}^{\prime} x_{2}^{3}-3 a \mathscr{B}^{\prime} x_{2}^{2} x_{3}-3 \mathbb{C}^{\prime} x_{2} x_{3}^{2}+\mathscr{B}^{\prime} x_{3}^{3}=0,
$$

and the equation of the Segre tangents is

$$
a^{2} \mathscr{B}^{\prime} x_{2}^{3}+3 a \mathbb{E}^{\prime} x_{2}^{2} x_{3}-3 a \mathscr{B}^{\prime} x_{2} x_{3}^{2}-\mathbb{C}^{\prime} x_{3}^{3}=0 .
$$

Finally, the equation (22) of the ray conic becomes

$$
\left(a \mathscr{B}^{\prime 2}+\mathfrak{C}^{\prime 2}\right)\left(a x_{2}^{2}+x_{3}^{2}\right)=a\left(x_{1}-\left(\mathbb{C}^{\prime} x_{2}-\mathscr{B}^{\prime} x_{3}^{2}\right)^{2} .\right.
$$

We shall now consider briefly the envelope which is analogous to the curve (35). If in equations (49) we replace $\lambda$ by $r \lambda$, we obtain the coördinates

- Lane, loc. cit., p. 286. 
of the ray point of $P_{y}$ with respect to the second family of the net $N_{\lambda h}$. The line coördinates of the ray of $P_{y}$ with respect to the net $N_{\lambda h}$ are then found to be given by the following formulas:

$$
\begin{aligned}
& u_{1}=r^{2} \lambda^{4} h^{4}+a\left(1+r^{2}\right) \lambda^{2} h^{2}+a^{2}, \\
& u_{2}=r \lambda^{2} h^{2}\left[-Q r \lambda^{2} h^{2}-a P(1+r) \lambda h+a Q\right], \\
& u_{3}=a\left[P r \lambda^{2} h^{2}-Q(1+r) \lambda h-a P\right] .
\end{aligned}
$$

The envelope of the ray, as $N_{\lambda h}$ varies over all the nets of the pencil determined by the net $N_{\lambda}$ in the bundle $B_{r}$, is therefore ordinarily a curve of class four. If, however, $r=-1$, and if $\lambda^{2} h^{2}+a \neq 0$, equations (54) reduce to

$$
u_{1}=\lambda^{2} h^{2}+a, \quad u_{2}=-Q \lambda^{2} h^{2}, \quad u_{3}=-a P,
$$

so that the result of eliminating $h$ is the linear relation

$$
P Q u_{1}+P u_{2}+Q u_{3}=0 \text {. }
$$

Therefore we have the following conclusion. If we consider a bundle of nets consisting of all nets every one of which has the property that at every surface point its tangents separate the tangents of a fundamental conjugate net harmonically, and if we consider a pencil of nets in this bundle, determined by a net $N_{\lambda}$ of the bundle, then the rays of a surface point $P_{y}$ with respect to all the nets of this pencil form a flat pencil of lines. If the fundamental conjugate net of the bundle is the parametric net of system (43), the center of the bundle is the point $(P Q, P, Q)$.

VIII. BUNDLES WITH NON-CONJUGATE NON-ASYMPTOTIC NETS FUNDAMENTAL

In a paper published* posthumously, G. M. Green employed the differential equations of a surface $S$, referred to a non-conjugate non-asymptotic net, in the form

$$
\begin{aligned}
& y_{u u}=a y_{u v}+b y_{u}+c y_{v}+d y, \\
& y_{v v}=a^{\prime} y_{u v}+b^{\prime} y_{u}+c^{\prime} y_{v}+d^{\prime} y .
\end{aligned}
$$

The differential equation of the asymptotic net in this notation is

$$
a d u^{2}+2 d u d v+a^{\prime} d v^{2}=0,
$$

and the family conjugate to a family $F_{\lambda}$, in these parameters, is given by

$$
(a+\lambda) d u+\left(a^{\prime} \lambda+1\right) d v=0 .
$$

\footnotetext{
- Green, Nets of space curves, these Transactions, vol. 21 (1920), pp. 207-236.
} 
If the parametric tangents form with the asymptotic tangents at every point of $S$ a constant cross ratio $r$, then equation (4) shows that we must have

$$
4 r=(1+r)^{2} a a^{\prime} .
$$

Therefore the parametric net on an integrating surface of system (55) belongs to the bundle determined by the asymptotic net and a constant cross ratio $r \neq-1$ if equation (58) is satisfied.

In studying the bundle determined by an arbitrary net not conjugate and not asymptotic, and a constant cross ratio $r$, we take the arbitrary net as the parametric net of system (55). Then, by a course of reasoning similar to that employed in the preceding section, we find that the ray point $\sigma_{\lambda}$ of a surface point $P_{y}$ with respect to the family $F_{\lambda}$, in arbitrary parameters, is given by the formula

$$
\sigma_{\lambda}=\left(\lambda^{2} a^{\prime}+2 \lambda+a\right) \rho+\lambda\left(\lambda^{2} a^{\prime}+2 \lambda+a\right) \sigma+\lambda(Q \lambda+P) y,
$$

wherein we have placed

$$
\begin{aligned}
& P=c a^{\prime}-c^{\prime} a-b-a \frac{b^{\prime}}{a^{\prime}}-2 \frac{c}{a}+\frac{\lambda_{u}}{\lambda}-a \frac{\lambda_{v}}{\lambda}, \\
& Q=b^{\prime} a-b a^{\prime}-c^{\prime}-a^{\prime} \frac{c}{a}-2 \frac{b^{\prime}}{a^{\prime}}-\frac{\lambda_{v}}{\lambda}+a^{\prime} \frac{\lambda_{u}}{\lambda},
\end{aligned}
$$

and $\rho, \sigma$ are the covariants defined by the formulas

$$
\rho=y_{u}+\frac{c}{a} y, \quad \sigma=y_{v}+\frac{b^{\prime}}{a^{\prime}} y .
$$

The points $\rho$ and $\sigma$ are the ray points of $P_{y}$ with respect to the curves $v=$ const. and the curves $u=$ const. respectively.

The ray point $\sigma_{\lambda h}$ of $P_{y}$ with respect to the first family of the net $N_{\lambda h}$ has coördinates which, referred to the local triangle $y, \rho, \sigma$ with suitably chosen unit point, are given by the following formulas:

$$
\begin{aligned}
& x_{1}=\lambda h(Q \lambda h+P), \\
& x_{2}=\lambda^{2} h^{2} a^{\prime}+2 \lambda h+a, \\
& x_{3}=\lambda h\left(\lambda^{2} h^{2} a^{\prime}+2 \lambda h+a\right) .
\end{aligned}
$$

Eliminating $h$ from these equations, we obtain

$$
\begin{aligned}
& \left(a x_{2}^{2}+2 x_{2} x_{3}+a^{\prime} x_{3}^{2}\right)\left[4\left(1-a a^{\prime}\right) x_{1}-(2 P-a Q) x_{2}-\left(2 Q-a^{\prime} P\right) x_{3}\right] \\
& \quad+a(2 P-a Q) x_{2}^{3}+3 a a^{\prime} P x_{2}^{2} x_{3}+3 a a^{\prime} Q x_{2} x_{3}^{2}+a^{\prime}\left(2 Q-a^{\prime} P\right) x_{3}^{3}=0 .
\end{aligned}
$$


This is the ray-point cubic of $P_{\nu}$ with respect to the system $G$ determined by the parametric net of system (55) and the family $F_{\lambda}$ in arbitrary parameters. It has a node at $P_{y}$, the double point tangents being the asymptotic tangents. It also has three inflexions which lie on the flex ray

$$
4\left(1-a a^{\prime}\right) x_{1}-(2 P-a Q) x_{2}-\left(2 Q-a^{\prime} P\right) x_{3}=0,
$$

and each infiexion lies on one of the lines through $P_{y}$,

$$
a(2 P-a Q) x_{2}^{3}+3 a a^{\prime} P x_{2}^{2} x_{3}+3 a a^{\prime} Q x_{2} x_{3}^{2}+a^{\prime}\left(2 Q-a^{\prime} P\right) x_{3}^{3}=0 .
$$

Let us suppose that the asymptotic net belongs to the system $G$ associated with the cubic (63). Then the cross ratio of the asymptotic tangents and the parametric tangents at every surface point is constant, so that $a a^{\prime}=$ const. Moreover, the cross ratio of the parametric tangents, the tangent belonging to the family $F_{\lambda}$, and the tangent of either family of asymptotic curves, at every surface point, is constant, so that

Then we find

$$
\lambda=\text { const. } \frac{-1+\sqrt{1-a a^{\prime}}}{a^{\prime}}=\frac{\text { const. }}{a^{\prime}} \text {. }
$$

$$
2 P-a Q=4 a \mathfrak{S}^{\prime(u)}, 2 Q-a^{\prime} P=4 a^{\prime} \mathfrak{S}^{\prime(v)},
$$

where $\mathfrak{C}^{\prime(u)}$ and $\mathfrak{C}^{\prime(v)}$ are the invariants* whose vanishing implies that the curves $C_{u}$ and $C_{v}$ respectively are Darboux curves. Under these conditions, if $a a^{\prime} \neq 4$, equation (63) becomes

$$
\begin{aligned}
& \left(a x_{2}^{2}+2 x_{2} x_{3}+a^{\prime} x_{3}^{2}\right)\left[\left(1-a a^{\prime}\right) x_{1}-a \mathfrak{S}^{{ }^{\prime(u)}} x_{2}-a^{\prime} \mathfrak{S}^{\prime(v)} x_{3}\right] \\
& +a^{2} \mathfrak{S}^{\prime(u)} x_{2}^{3}-\frac{3 a^{2} a^{\prime}}{a a^{\prime}-4}\left(2 \mathfrak{S}^{\prime(u)}+a^{\prime} \mathfrak{S}^{\prime(v)}\right) x_{2}^{2} x_{3} \\
& \quad-\frac{3 a a^{\prime 2}}{a a^{\prime}-4}\left(a \mathfrak{S}^{\prime(u)}+2 \mathfrak{S}^{\prime(v)}\right) x_{1} x_{3}^{2}+a^{\prime 2} \mathfrak{S}^{\prime(v)} x_{2}^{3}=0 .
\end{aligned}
$$

This is the ray-point cubic of the system $G$ which is determined by the asymptotic net and one family of the parametric curves of system (55), and which also contains the other parametric family. If $a a^{\prime}=4$, equation (58) shows that the cross ratio of the parametric tangents and asymptotic tangents at every surface point is a root of the equation $1+r+r^{2}=0$.

\footnotetext{
* Green, Nets of space curves, p. 217.
} 
The line coördinates of the ray of $P_{y}$ with respect to the net $N_{\lambda h}$ represented by equation (9) in arbitrary parameters are found to be given by the following equations, analogous to equations (54):

$$
\begin{aligned}
& u_{1}=r^{2} a^{\prime 2} \lambda^{4} h^{4}+2 a^{\prime} r(1+r) \lambda^{3} h^{3}+\left(4 r+a a^{\prime}+a a^{\prime} r^{2}\right) \lambda^{2} h^{2}+2 a(1+r) \lambda h+a^{2}, \\
& u_{2}=-r \lambda^{2} h^{2}\left[a^{\prime} Q r \lambda^{2} h^{2}+a^{\prime} P(1+r) \lambda h+2 P-a Q\right], \\
& u_{3}=-\left(2 Q-a^{\prime} P\right) r \lambda^{2} h^{2}-a Q(1+r) \lambda h-a P,
\end{aligned}
$$

where $P$ and $Q$ are the functions defined in equations (60). The envelope of this ray as the net $N_{\lambda h}$ varies over all the nets of the pencil determined by the net $N_{\lambda}$ in the bundle $B_{r}$ is, therefore, ordinarily of class four. However, if $r=-1$, equations (66) reduce to

$$
\begin{aligned}
& u_{1}=a^{\prime 2} \lambda^{4} h^{4}+2\left(a a^{\prime}-2\right) \lambda^{2} h^{2}+a^{2}, \\
& u_{2}=\lambda^{2} h^{2}\left(-a^{\prime} Q \lambda^{2} h^{2}+2 P-a Q\right), \\
& u_{3}=\left(2 Q-a^{\prime} P\right) \lambda^{2} h^{2}-a P
\end{aligned}
$$

and the envelope is a conic.

University of Chicago,

Chicago, InL. 